\title{
Estimating Nonlinear Systems in a Neighborhood of LTI-approximants
}

\author{
Martin Enqvist, Lennart Ljung \\ Division of Automatic Control \\ Department of Electrical Engineering \\ Linköpings universitet, SE-581 83 Linköping, Sweden \\ WWW: http://wWw. control.isy.liu.se \\ E-mail: maren,ljung@isy.liu.se
}

August 26, 2002

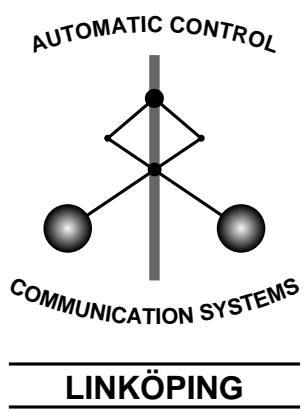

Report no.: LiTH-ISY-R-2459

Submitted to CDC 2002, Las Vegas, USA

Technical reports from the Control \& Communication group in Linköping are available at http://www.control.isy.liu.se/publications. 



\title{
Estimating Nonlinear Systems in a Neighborhood of LTI-approximants
}

\author{
Martin Enqvist, Lennart Ljung
}

August 26, 2002

\begin{abstract}
The estimation of Linear Time Invariant (LTI) models is a standard procedure in System Identification. Any real-life system will however be nonlinear and time-varying, and the estimated model will converge to the LTI second order equivalent (LTI-SOE) of the true system. In this paper we consider some aspects of this convergence and the distance between the true system and its LTI-SOE. We show that there may be cases where even the slightest nonlinearity may cause big differences in the LTI-SOE. We also show a result that gives conditions that guarantee that the LTISOE is close to "the natural" LTI approximant. Finally, an upper bound on the distance between the LTI-SOE of a nonlinear FIR system with a white input signal and the linear part of the system is derived.
\end{abstract}

\section{LTI Model Identification}

To estimate Linear Time Invariant (LTI) models from observed data is a standard tool in systems and control, see e.g. [1]. A brief summary of the basic procedure is as follows:

A general LTI-model of a dynamical system can always be described as

$$
y(t)=G(q, \theta) u(t)+H(q, \theta) e(t)
$$

Here, $q$ is the shift operator, and $G$ and $H$ are the transfer matrices from the measured input $u$ and the noise source $e$, which is modeled as white noise (sequence of independent random variables). For notational convenience we will from now on only consider Single-Input-Single-Output systems, but the theory is the same in the multi-variable case.

The transfer functions are parameterized by a finite-dimensional parameter vector $\theta$, and this parameterization can be quite arbitrary. For black-box models, it is common to parameterize $G$ and $H$ in terms of the coefficients of numerator and denominator polynomials, perhaps constraining $G$ and $H$ to have the same denominators. This leads to well established model classes, known under names like ARX, ARMAX, OE, BJ, etc. The model parameterizations could also correspond to state-space models in discrete or continuous time.

Whatever the parameterization, the problem is to estimate the parameters in (1) based on observed input-output sequences $\{y(t), u(t), t=1,2, \ldots, N\}$. 
A common approach is formed by Prediction error methods that first determine the prediction errors associated with (1):

$$
\varepsilon(t, \theta)=H^{-1}(q, \theta)(y(t)-G(q, \theta) u(t))
$$

This requires $\theta$ be confined to a region $D$, so that the filters $H^{-1}$ and $H^{-1} G$ are stable. Then the $\theta$ that minimizes the norm of the errors

$$
\begin{aligned}
\hat{\theta}_{N} & =\arg \min _{\theta \in D} V_{N}(\theta) \\
V_{N}(\theta) & =\frac{1}{N} \sum_{t=1}^{N} \varepsilon^{2}(t, \theta)
\end{aligned}
$$

is determined, typically by numerical search.

How will these methods perform? Well, that depends on the input-output data. A typical approach to analysis is to assume that the data indeed have been generated by a system like (1) for some particular parameter vector $\theta_{0}$, and for $e$ being a sequence of independent random variables. In that case the asymptotic statistical properties (convergence and asymptotic distribution) of $\hat{\theta}_{N}$ can be calculated readily. We refer to [1] for an analysis of this kind, as well as for more details on model structures and estimation techniques.

\section{Estimating LTI Models of Nonlinear Systems}

The question we discuss in this paper is what happens with the model $\hat{\theta}_{N}$ in case the data originate from a non-LTI system. The use of linear models of nonlinear systems can be discussed in several different frameworks and related material can be found e.g. in [3], [4], [5] and [6]. In this paper we will consider input and output signals that are jointly quasi-stationary (cf. [1]) and that have well-defined spectral densities according to the following definition.

Definition 2.1 A signal $s(t)$ is said to be quasi-stationary if it is subject to

$$
\begin{aligned}
& \mathrm{E}\{s(t)\}=m_{s}(t), \quad\left|m_{s}(t)\right| \leq C \quad \forall t \\
& \mathrm{E}\{s(t) s(r)\}=R_{s}(t, r), \quad\left|R_{s}(t, r)\right| \leq C \quad \forall t, r \\
& \lim _{N \rightarrow \infty} \frac{1}{N} \sum_{t=1}^{N} R_{s}(t, t-\tau)=R_{s}(\tau), \quad \forall \tau \\
& \sum_{\tau=-\infty}^{\infty}\left|R_{s}(\tau)\right|<+\infty
\end{aligned}
$$

Two signals $u(t)$ and $y(t)$ are said to be jointly quasi-stationary if they, in addition to being quasi-stationary by themselves, are subject to

$$
\begin{aligned}
& \mathrm{E}\{y(t) u(r)\}=R_{y u}(t, r), \quad\left|R_{y u}(t, r)\right| \leq C \quad \forall t, r \\
& \lim _{N \rightarrow \infty} \frac{1}{N} \sum_{t=1}^{N} R_{y u}(t, t-\tau)=R_{y u}(\tau), \quad \forall \tau \\
& \sum_{\tau=-\infty}^{\infty}\left|R_{y u}(\tau)\right|<+\infty
\end{aligned}
$$


Let $\Phi_{u}(z)$ and $\Phi_{y u}(z)$ denote the $z$-transforms of $R_{u}(\tau)$ and $R_{y u}(\tau)$, respectively.

$$
\begin{gathered}
\Phi_{u}(z)=\sum_{\tau=-\infty}^{\infty} R_{u}(\tau) z^{-\tau} \\
\Phi_{y u}(z)=\sum_{\tau=-\infty}^{\infty} R_{y u}(\tau) z^{-\tau}
\end{gathered}
$$

The spectral densities $\Phi_{u}\left(e^{i \omega}\right)$ and $\Phi_{y u}\left(e^{i \omega}\right)$ will then be well-defined for all $\omega \in[-\pi, \pi]$.

Note that the class of quasi-stationary signals contains both purely stochastic and purely deterministic signals as well as signals that have both stochastic and deterministic components.

The basic result that is used in our context is as follows (cf. [1] and [2]). Suppose that the input-output signals fulfill the requirements in Definition 2.1 and that the model (1) is an output error model, i.e. that $H(q, \theta)=1$. Then, as $N \rightarrow \infty$

$$
\hat{\theta}_{N} \rightarrow \arg \min _{\theta} \int_{-\pi}^{\pi}\left\|G\left(e^{i \omega}, \theta\right)-G_{0}\left(e^{i \omega}\right)\right\|^{2} \Phi_{u}\left(e^{i \omega}\right) d \omega
$$

where

$$
G_{0}\left(e^{i \omega}\right)=\left[\frac{\Phi_{y u}\left(e^{i \omega}\right)}{\Phi_{u}\left(e^{i \omega}\right)}\right]_{\text {causal }}
$$

and where $[\ldots]_{\text {causal }}$ denotes taking the causal part.

The convergence theory is thus rather straightforward, and we shall in the following section investigate how $G_{0}$, the LTI Second Order Equivalent (LTI$\mathrm{SOE}$ ) depends on the true underlying system and the input properties.

Before that, let us however note a few special features of the convergence to the LTI-SOE:

- Even if the true system is causal, the ratio $\Phi_{y u} / \Phi_{u}$ may correspond to a non-causal function, so taking the causal part in (5) is essential. This resembles the situation in linear systems, when output feedback is present.

- Even if the data from the system is noise-free, the convergence of the estimates will exhibit "stochastic features": The LTI-SOE will be approached typically with the rate $1 / \sqrt{N}$ and the path taken to the limit will depend on the actual realization of the input.

\section{Properties of LTI-SOE:s for Almost Linear Sys- tems}

The use of a linear model is very natural when the true system is close to being linear. In many cases, the behavior of an almost linear system can be understood, at least intuitively, from the theory of linear systems. Hence, it is a legitimate question to ask whether this linear intuition can be extended also to LTI-SOE:s for almost linear systems. 
For example, if the nonlinear contribution to the output is small for a certain input one might assume that the corresponding LTI-SOE would be close to the linear part of the system in some sense. However, as we will see in the following example, this is not always the case.

\section{Example 3.1: The distance between the LTI-SOE and the linear part of a system}

Consider the system

$$
\begin{aligned}
y(t) & =u(t)+0.4 u(t-1)+\alpha\left(\frac{3}{4} u(t-1)-u^{3}(t-1)\right) \\
& =G_{L}(q) u(t)+\alpha h(u(t-1))
\end{aligned}
$$

where $G_{L}(q)=1+0.4 q^{-1}$ and where $h$ is a static nonlinearity with $h(x)=$ $\frac{3}{4} x-x^{3}$. The parameter $\alpha$ defines how close the system is to the linear system $G_{L}$. For a bounded input, small values of $\alpha$ will give a system output that is close to the output from $G_{L}$. Assume that the input signal is

$$
u(t)=\sin (0.1 t)+\varepsilon \sin (0.3 t)
$$

where $\varepsilon=0.001$. For this input, a small value of $\alpha$, like for example $\alpha=0.01$, will give an output that is very similar to the output from $G_{L}$ (i.e. the output when $\alpha=0)$.

The small differences between these output signals will however give rise to totally different LTI-SOE:s. This can be seen if we estimate two output error models with $n_{f}=n_{b}=2$, and $n_{k}=0$ (cf. [1]). The parameters of these models have been estimated from two data sets consisting of 10000 noise-free input output measurements with $\alpha=0$ and $\alpha=0.01$, respectively. The estimated model $G_{1}$ that is obtained when $\alpha=0$ is of course equal to $G_{L}$. This is however not the case for the model estimate $G_{2}$ that is obtained for $\alpha=0.01$. Figure 1 shows the differences between $G_{L}, G_{1}$ and $G_{2}$ in the frequency domain.

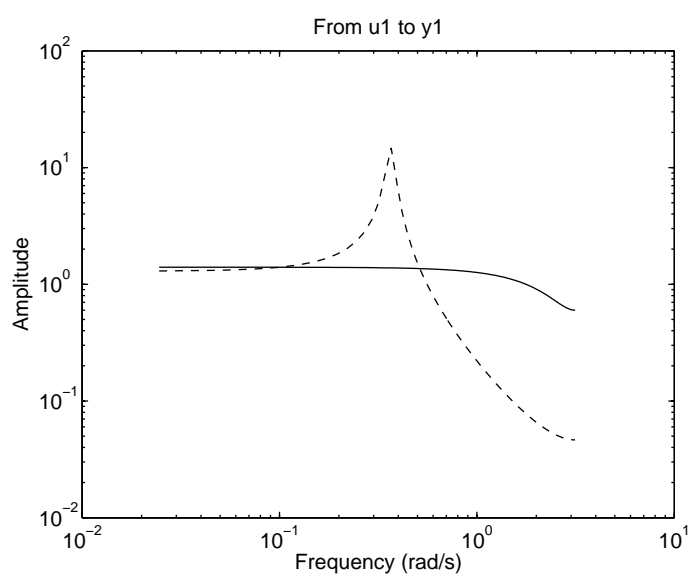

Figure 1: The frequency responses of $G_{L}=G_{1}$ (solid) and $G_{2}$ (dashed).

If we use many measurements we know from (4) that the estimated LTI model will approximate the LTI-SOE of the system for this particular input. 
The estimated models will be as close to the LTI-SOE:s as possible at the frequencies 0.1 and $0.3 \mathrm{rad} / \mathrm{s}$. Thus we can conclude from Figure 1 that the two systems (with $\alpha=0$ and $\alpha=0.01$ ) have very different LTI-SOE:s.

The dramatic change in the estimated LTI model that can be seen when a small nonlinearity is introduced is a clear indication on that the LTI-SOE not always can be understood from linear theory. It should be noted that a small linear, time-invariant deviation from $G_{L}$ only would have given rise to a small deviation in the estimated LTI model as an LTI system cannot generate harmonics.

In our example, no matter how small $\alpha$ we choose we can always choose an even smaller $\varepsilon$ in $u$ and thus get an LTI-SOE far from $G_{L}$. That is, no matter how linear we make the system there is always a $u$ that have an LTI-SOE far from $G_{L}$.

In the previous example we have seen that the LTI-SOE in some cases can be far from the linear part of the system. Let $u_{-\infty}^{t}$ denote the set of input signals from time $-\infty$ to time $t$. Consider a system $y(t)=f\left(u_{-\infty}^{t}, \alpha\right)$ that has a quasi-stationary output and where $\alpha$, just like in the previous example, is a parameter that defines the size of the nonlinear part of $f$. Assume that $f\left(u_{-\infty}^{t}, \alpha\right) \rightarrow f\left(u_{-\infty}^{t}, 0\right)$ when $\alpha \rightarrow 0 \forall t \in \mathbb{Z}$ and for all quasi-stationary $u$. Assume further that $f\left(u_{-\infty}^{t}, 0\right)$ is a stable, causal LTI system $G_{L}$, i.e.

$$
f\left(u_{-\infty}^{t}, 0\right)=\sum_{k=0}^{\infty} g_{L}(k) u(t-k)=G_{L}(q) u(t)
$$

Let $G_{0, \alpha, u}$ denote the LTI-SOE that is obtained for a certain input signal $u$ and a certain $\alpha$.

The conclusion that we can draw from Example 3.1 is that we cannot in general assume that

$$
\sup _{u: u q . s .} \int_{-\pi}^{\pi}\left|G_{0, \alpha, u}\left(e^{i \omega}\right)-G_{L}\left(e^{i \omega}\right)\right| d \omega
$$

will approach 0 when $\alpha \rightarrow 0$. (The supremum is taken over all quasi-stationary $u$ ). For some systems we can, whenever there is a small nonlinear term in the system output, find a $u$ for which the LTI-SOE is far from $G_{L}$.

On the other hand, in cases where the nonlinear parts of the system are more significant, the LTI-SOE will be a much better model of the system for signals that are similar to the signal that was used to generate the LTI-SOE.

Despite the fact that we even for an almost linear system cannot prove that the LTI-SOE:s are close to the linear part of the system for all inputs it is often possible to say something about the behavior of the LTI-SOE for a particular input signal. As a matter of fact, for a fixed input signal and a nonlinear system that fulfill some additional requirements we have the following theorem.

Theorem 3.1 Let $y(t)=f\left(u_{-\infty}^{t}, \alpha\right)$ be a nonlinear system and let $u$ be a given deterministic sequence that is quasi-stationary. Let $R_{y u, \alpha}(\tau)$ be the cross covariance function between $y$ and $u$ for a certain choice of $\alpha$ and let $\Phi_{y u, \alpha}(z)$ be the $z$-transform of $R_{y u, \alpha}(\tau)$. Furthermore, let $G_{0, \alpha, u}$ denote the LTI-SOE of the system for a particular choice of $\alpha$. Assume that the following holds 
(i) $u(t)$ is such that $\int_{-\pi}^{\pi} \frac{1}{\Phi_{u}\left(e^{i \omega}\right)^{2}} d \omega=I_{u}<+\infty$ and that $G_{0,0, u}=G_{L}$.

(ii) $y(t)$ is such that it, together with $u$, fulfills the conditions in Definition 2.1 for every choice of $\alpha$ with $|\alpha|<\alpha_{\max }$.

(iii) $\alpha=0$ gives a stable, causal LTI system $f\left(u_{-\infty}^{t}, 0\right)=\sum_{k=0}^{\infty} g_{L}(k) u(t-k)=$ $G_{L}(q) u(t)$ and $f\left(u_{-\infty}^{t}, \alpha\right) \rightarrow f\left(u_{-\infty}^{t}, 0\right), \alpha \rightarrow 0$ pointwise for each $t \in \mathbb{Z}$.

(iv) $\exists M_{u} \in \mathbb{Z}_{+}, \lambda_{u}, 0 \leq \lambda_{u}<1, K_{u}>0$ such that $\left|R_{y u, \alpha}(\tau)\right|<K_{u} \lambda_{u}^{\tau}$ when $\tau>M_{u} \forall \alpha$ with $|\alpha|<\alpha_{\max }$.

Then it follows that

$$
\int_{-\pi}^{\pi}\left|G_{0, \alpha, u}\left(e^{i \omega}\right)-G_{L}\left(e^{i \omega}\right)\right| d \omega \rightarrow 0, \quad \alpha \rightarrow 0
$$

Proof: First we want to show that $R_{y u, \alpha} \rightarrow R_{y u, 0}$. Take an arbitrary $\varepsilon_{1}>0$.

$$
\begin{aligned}
& \left|R_{y u, \alpha}(\tau)-R_{y u, 0}(\tau)\right| \leq\left|R_{y u, \alpha}(\tau)-\frac{1}{N_{0}} \sum_{t=1}^{N_{0}} f\left(u_{-\infty}^{t}, \alpha\right) u(t-\tau)\right|+ \\
& +\frac{1}{N_{0}} \sum_{t=1}^{N_{0}}\left|f\left(u_{-\infty}^{t}, \alpha\right)-f\left(u_{-\infty}^{t}, 0\right)\right| \sup |u(t)|+ \\
& +\left|\frac{1}{N_{0}} \sum_{t=1}^{N_{0}} f\left(u_{-\infty}^{t}, 0\right) u(t-\tau)-R_{y u, 0}(\tau)\right|
\end{aligned}
$$

Choose $N_{0}$ such that the sum of the first and the third term above is less than $2 \varepsilon_{1} / 3$. Then $\exists \delta_{\varepsilon_{1}}>0$ such that $|\alpha|<\delta_{\varepsilon_{1}} \Rightarrow \max _{1 \leq t \leq N_{0}} \mid f\left(u_{-\infty}^{t}, \alpha\right)-$ $f\left(u_{-\infty}^{t}, 0\right) \mid<\frac{\varepsilon_{1}}{3 \sup |u(t)|}$. Thus $\left|R_{y u, \alpha}(\tau)-R_{y u, 0}(\tau)\right|<\varepsilon_{1}$ if $|\alpha|<\delta_{\varepsilon_{1}}$, i.e.

$$
R_{y u, \alpha}(\tau) \rightarrow R_{y u, 0}(\tau), \quad \alpha \rightarrow 0
$$

We continue by proving that $\int_{-\pi}^{\pi}\left|\Phi_{y u, \alpha}\left(e^{i \omega}\right)-\Phi_{y u, 0}\left(e^{i \omega}\right)\right|^{2} d \omega \rightarrow 0$. Take an arbitrary $\varepsilon_{2}>0$. By Parseval's identity we get

$$
\begin{aligned}
& \int_{-\pi}^{\pi}\left|\Phi_{y u, \alpha}\left(e^{i \omega}\right)-\Phi_{y u, 0}\left(e^{i \omega}\right)\right|^{2} d \omega=2 \pi \sum_{\tau=-\infty}^{\infty}\left|R_{y u, \alpha}(\tau)-R_{y u, 0}(\tau)\right|^{2} \leq \\
& 2 \pi \sum_{\tau=-C_{0}}^{C_{0}}\left|R_{y u, \alpha}(\tau)-R_{y u, 0}(\tau)\right|^{2}+4 \pi \sum_{\tau=C_{0}+1}^{\infty}\left(\left|R_{y u, \alpha}(\tau)\right|^{2}+\left|R_{y u, 0}(\tau)\right|^{2}+\right. \\
& \left.+2\left|R_{y u, \alpha}(\tau)\right|\left|R_{y u, 0}(\tau)\right|\right)
\end{aligned}
$$

Choose $C_{0}$ such that the last sum is less than $\varepsilon_{2} / 2 \forall \alpha$ with $|\alpha|<\alpha_{\max }$. (This is possible according to assumption (iv)). Then, from the first part of the proof, it follows that $\exists \delta_{\varepsilon_{2}}>0$ such that $|\alpha|<\delta_{\varepsilon_{2}} \Rightarrow 2 \pi \sum_{\tau=-C_{0}}^{C_{0}}\left|R_{y u, \alpha}(\tau)-R_{y u, 0}(\tau)\right|^{2}<$ $\varepsilon_{2} / 2$. Thus $\int_{-\pi}^{\pi}\left|\Phi_{y u, \alpha}\left(e^{i \omega}\right)-\Phi_{y u, 0}\left(e^{i \omega}\right)\right|^{2} d \omega<\varepsilon_{2}$ if $|\alpha|<\delta_{\varepsilon_{2}}$, i.e.

$$
\int_{-\pi}^{\pi}\left|\Phi_{y u, \alpha}\left(e^{i \omega}\right)-\Phi_{y u, 0}\left(e^{i \omega}\right)\right|^{2} d \omega \rightarrow 0, \quad \alpha \rightarrow 0
$$


Schwarz' inequality now gives

$$
\begin{aligned}
& \int_{-\pi}^{\pi}\left|G_{0, \alpha, u}\left(e^{i \omega}\right)-G_{L}\left(e^{i \omega}\right)\right| d \omega=\int_{-\pi}^{\pi} \frac{\left|\Phi_{y u, \alpha}\left(e^{i \omega}\right)-\Phi_{y u, 0}\left(e^{i \omega}\right)\right|}{\Phi_{u}\left(e^{i \omega}\right)} d \omega \leq \\
& \left(\int_{-\pi}^{\pi}\left|\Phi_{y u, \alpha}\left(e^{i \omega}\right)-\Phi_{y u, 0}\left(e^{i \omega}\right)\right|^{2} d \omega\right)^{1 / 2} \cdot\left(\int_{-\pi}^{\pi} \frac{1}{\Phi_{u}\left(e^{i \omega}\right)^{2}} d \omega\right)^{1 / 2}= \\
& \left(\int_{-\pi}^{\pi}\left|\Phi_{y u, \alpha}\left(e^{i \omega}\right)-\Phi_{y u, 0}\left(e^{i \omega}\right)\right|^{2} d \omega\right)^{1 / 2} \cdot I_{u}^{1 / 2}
\end{aligned}
$$

and the result (10) follows.

(N.B. Assumption (iv) can as a matter of fact be relaxed a bit. The important thing is that $R_{y u, \alpha}(\tau)$ is small enough for large $\tau$ independently of $\alpha$.)

Theorem 3.1 gives conditions on the system and input that guarantee a continuous behavior of the LTI-SOE of the system in the point where $\alpha=0$, i.e. when the system is linear. This is of course not surprising, it is rather the kind of behavior one would expect the system to possess. Hence, the interesting part of the theorem is rather the conditions that are required to prove the result than the result itself.

The previous theorem tells us that the LTI-SOE will converge towards the linear part of the system when the nonlinearity tends to zero but not how fast this convergence is. In order to be able to derive an upper bound on the distance between the LTI-SOE and the linear part of a system with a nonzero nonlinearity of a certain size we will have to make some new restrictions on the types of systems and excitation signals.

Hence, we will from now on only consider nonlinear FIR systems with white stochastic inputs that can be written like $y(t)=f\left(u_{t-M}^{t}\right)$ and that are close to a linear system $z(t)=\sum_{k=0}^{M} g_{L}(k) u(t-k)$. The following theorem gives an upper bound on the distance between the LTI-SOE and the linear part of such a nonlinear system.

Theorem 3.2 Let $u(t)$ be a quasi-stationary sequence of independent random variables with zero mean and let $y(t)=f\left(u_{t-M}^{t}\right)$ be a nonlinear FIR system such that

$$
\left|f\left(u_{t-M}^{t}\right)-\sum_{k=0}^{M} g_{L}(k) u(t-k)\right|<a
$$

Assume that the output $y(t)$ has zero mean and that it, together with $u(t)$, fulfills the conditions in Definition 2.1. Assume also that $\lim _{N \rightarrow \infty} \frac{1}{N} \sum_{t=1}^{N} \mathrm{E}\{|u(t)|\}<$ $+\infty$.

Then

$$
\begin{aligned}
& \int_{-\pi}^{\pi}\left|G_{0}\left(e^{i \omega}\right)-G_{L}\left(e^{i \omega}\right)\right| d \omega< \\
& a 2 \pi \sqrt{(M+1)}\left(\frac{\lim _{N \rightarrow \infty} \frac{1}{N} \sum_{t=1}^{N} \mathrm{E}\{|u(t)|\}}{\lim _{N \rightarrow \infty} \frac{1}{N} \sum_{t=1}^{N} \mathrm{E}\left\{u(t)^{2}\right\}}\right)
\end{aligned}
$$


Proof: We start by proving the following inequality

$$
\begin{aligned}
& \left|R_{y u}(\tau)-\sum_{k=0}^{M} g_{L}(k) R_{u}(\tau-k)\right|<a \lim _{N \rightarrow \infty} \frac{1}{N} \sum_{t=1}^{N} \mathrm{E}\{|u(t-\tau)|\} \\
& \left|R_{y u}(\tau)-\sum_{k=0}^{M} g_{L}(k) R_{u}(\tau-k)\right|=\mid \lim _{N \rightarrow \infty} \frac{1}{N} \sum_{t=1}^{N} \mathrm{E}\{y(t) u(t-\tau)\}- \\
& -\sum_{k=0}^{M} g_{L}(k) \lim _{N \rightarrow \infty} \frac{1}{N} \sum_{t=1}^{N} \mathrm{E}\{u(t-k) u(t-\tau)\} \mid= \\
& \lim _{N \rightarrow \infty}\left|\frac{1}{N} \sum_{t=1}^{N} \mathrm{E}\left\{\left(y(t)-\sum_{k=0}^{M} g_{L}(k) u(t-k)\right) u(t-\tau)\right\}\right| \leq \\
& \lim _{N \rightarrow \infty} \frac{1}{N} \sum_{t=1}^{N} \mathrm{E}\left\{\left|y(t)-\sum_{k=0}^{M} g_{L}(k) u(t-k)\right||u(t-\tau)|\right\}< \\
& a \lim _{N \rightarrow \infty} \frac{1}{N} \sum_{t=1}^{N} \mathrm{E}\{|u(t-\tau)|\}
\end{aligned}
$$

The assumption that $u(t)$ consists of independent random variables implies that $\Phi_{u}\left(e^{i \omega}\right)=R_{u}(0)$ and that $R_{y u}(\tau)=0$ when $\tau>M$ or $\tau<0$. This, together with Parseval's identity and equation (13) give

$$
\begin{aligned}
& \int_{-\pi}^{\pi}\left|G_{0}\left(e^{i \omega}\right)-G_{L}\left(e^{i \omega}\right)\right|^{2} d \omega= \\
& \frac{1}{R_{u}(0)^{2}} \int_{-\pi}^{\pi}\left|\Phi_{y u}\left(e^{i \omega}\right)-G_{L}\left(e^{i \omega}\right) R_{u}(0)\right|^{2} d \omega= \\
& \frac{2 \pi}{R_{u}(0)^{2}} \sum_{\tau=0}^{M}\left|R_{y u}(\tau)-g_{L}(\tau) R_{u}(0)\right|^{2}< \\
& a^{2} 2 \pi(M+1)\left(\frac{\lim _{N \rightarrow \infty} \frac{1}{N} \sum_{t=1}^{N} \mathrm{E}\{|u(t)|\}}{\lim _{N \rightarrow \infty} \frac{1}{N} \sum_{t=1}^{N} \mathrm{E}\left\{u(t)^{2}\right\}}\right)^{2}
\end{aligned}
$$

Finally, Schwarz' inequality gives

$$
\begin{aligned}
& \int_{-\pi}^{\pi}\left|G_{0}\left(e^{i \omega}\right)-G_{L}\left(e^{i \omega}\right)\right| d \omega \leq \\
& \left(\int_{-\pi}^{\pi}\left|G_{0}\left(e^{i \omega}\right)-G_{L}\left(e^{i \omega}\right)\right|^{2} d \omega\right)^{1 / 2} \sqrt{2 \pi}< \\
& a 2 \pi \sqrt{(M+1)}\left(\frac{\lim _{N \rightarrow \infty} \frac{1}{N} \sum_{t=1}^{N} \mathrm{E}\{|u(t)|\}}{\lim _{N \rightarrow \infty} \frac{1}{N} \sum_{t=1}^{N} \mathrm{E}\left\{u(t)^{2}\right\}}\right)
\end{aligned}
$$

Theorem 3.2 tells us that the distance between the LTI-SOE and $G_{L}$ is less than a bound that is proportional to $a$ (where $a$ is the size of the nonlinearity 
in equation (11)). Furthermore, this theorem shows the effect on the LTI-SOE of a scaling of the input signal.

The use of $\tilde{u}=c u$ as input instead of $u$ will result in a new LTI-SOE. The distance between this new LTI-SOE and $G_{L}$ will have an upper bound in equation (12) that is $\frac{1}{|c|}$ times the original bound. When a white input signal is used it is thus possible to reduce the distance between the LTI-SOE and the linear part of a nonlinear FIR system that fulfill equation (11) simply by scaling the input signal.

\section{Conclusions}

It is an important task in system identification to understand how general systems are approximated by LTI models. This includes the problem how to decide the LTI-SOE and to assess its "distance" to the true, nonlinear system. This task is technically difficult, and in this paper we have only investigated the "skin" of the set of LTI models in the set of general systems. We have shown that an LTI-SOE of an almost linear system can be far from the linear part of the system for some inputs. Furthermore, we have given conditions on the system and input that guarantee that the LTI-SOE approaches the linear part of the system when the nonlinear elements of the system approach zero. We have also derived an upper bound on the distance between the LTI-SOE of a nonlinear FIR system with a white input signal and the linear part of the system.

\section{Acknowledgments}

This work has been supported by the Swedish Research Council, which is hereby gratefully acknowledged.

\section{References}

[1] L. Ljung. System Identification - Theory for the User. Prentice-Hall, Upper Saddle River, N.J., 2nd edition, 1999.

[2] L. Ljung. Estimating linear time invariant models of non-linear time-varying systems. European Journal of Control, 7(2-3):203-219, Sept 2001. Semiplenary presentation at the European Control Conference, Sept 2001.

[3] P.M. Mäkilä and J.R. Partington. Linear models for nonlinear systems. To appear, Private Communication, July 2001.

[4] J.R. Partington and P.M. Mäkilä. On system gains for linear and nonlinear systems. To appear, Private Communication, December 2001.

[5] R. Pintelon and J. Schoukens. System Identification - A Frequency Domain Approach. IEEE Press, 2001.

[6] M. Schetzen. The Volterra and Wiener Theories of Nonlinear Systems. John Wiley \& Sons, 1980. 